\title{
Contaminación sonora por ruido vehicular en la Avenida Javier Prado
}

\author{
Recepción: Febrero de 2007 / Aceptación: Mayo de 2007
}

(1) Eulogio Santos De La Cruz

\begin{abstract}
RESUMEN
La contaminación sonora producida por el ruido de los vehículos es el factor que más molestias causa a la población urbana, los habitantes de Lima están expuestos a este problema, esto implica conocer la problemática del ruido y que demanda un ingente esfuerzo, resultando difícil abarcar toda la ciudad, por ello se opta por zonas, eligiendo la avenida Javier Prado, entre la intersección con la avenida Aviación por el Este y la avenida Brasil por el Oeste, haciendo encuestas a los transeúntes y conductores en la hora pico 07:00-09:00 y 15:00-19:00 horas en el área de más densa congestión vehicular, para mitigar se requiere una buena planificación urbana, diseño medioambiental óptimo de las vías y con el adecuado uso del suelo se lograría un mínimo impacto del ruido.
\end{abstract}

Palabras clave: Contaminación sonora, concentración, aprendizaje.

SONOROUS POLLUTION BY VEHICLE IN JAVIER PRADO AVENUE

\section{ABSTRACT}

The sonorous pollution produced by the noise of vehicles is the factor that more annoyances causes to the urban population, the in habitants of Lima are exposed to this problem, it implies to know the problematic of the noise and it demands an enormous effort, being difficult to include all the city, for that reason it's focused on zones, choosing Javier Prado avenue, between the intersection with the Aviacion avenue by the East and the Brazil avenue by the West, making surveys to the passers-by and conductors in the rush hour 07:00 - 09:00 and 15:00 - 19: 00 hours in the dense area of congestion to vehicular, to mitigate requires a good urban planning, optimal environmental design of ways and with the suitable use of the ground will get a minimum impact of the noise.

Key words: Sonorous pollution, concentration, learning.

\section{INTRODUCCIÓN}

Sobre el problema del medio ambiente y su deterioro es referirnos al comportamiento de los ciudadanos y la actitud pasiva de las autoridades que conjuntamente ejercen una acción destructiva, las distintas formas de la destrucción del medio ambiente son las contaminaciones por la explotación de los recursos naturales sin programas adecuados, eliminación de las aguas servidas a los ríos y el mar, en el caso de la ciudad de Lima el crecimiento vertiginoso en los últimos años del parque automotor estimulado por una disposición que facilita la importación de vehículos usados, su efecto inmediato es la contaminación del aire por los gases particulados liberados por estos vehículos, tanto particulares como de servicio público, los conductores hacen uso y abuso de claxon, sirenas y otras formas de producir el ruido, causando que la contaminación por este medio (el ruido) adquiera un asunto preocupante por su efecto en la salud y la conducta de los ciudadanos.

Por tratarse de una ciudad como Lima una gran metrópoli, se ha seleccionado a la avenida Javier Prado por ser una de las vías principales de la capital de la República y de más densa congestión vehicular, por donde convergen conductores y transeúntes, llegando en las llamadas horas pico que son entre 07:00-09:00 y 15:00-19:00 horas, un flujo en ambos sentidos de unos 10000 vehículos por hora, notándose que un vehículo avanza a un promedio de $3 \mathrm{~km} / \mathrm{h}$, cuando lo normal es de $45 \mathrm{~km} / \mathrm{h}$, igualmente cruzan la vía alrededor de 6000 personas por hora, por consiguiente en esta vía las condiciones de sonido normales son alteradas con el grave perjuicio para la salud de las personas y la economía.

Es pertinente destacar que desde aproximadamente cincuenta años, la comunidad científica mundial ha mostrado interés creciente en el estudio de la contaminación ambiental, de modo particular el ruido como contaminante ambiental por que tiene efectos perjudiciales sobre las personas expuestas, los especialistas coinciden en afirmar que el ruido es actualmente uno de los agentes contaminantes más generalizados que existen en todos los países industrializados. En el Perú adquiere mayor énfasis a partir de los años noventa, cuando el gobierno de ese entonces toma interés y promulga el Código de Medio Ambiente, precisando acerca de la contaminación ambiental y las responsabilidades de los agentes generadores.

\section{ACERCADELRUIDO}

Las informaciones recientes señalan que Lima, es una de las ciudades con mayor crecimiento de agentes productoras de sonidos y ruidos,

(1) Magíster en Ciencias. Profesor del Departamento de Diseño y Tecnología Industrial, UNMSM.
E-mail: esantosd@unmsm.edu.pe 
éstos tienen una variedad, intensidad y perdurabilidad, por tanto constituyen una forma de contaminación física.

Según reporte de la Organización Mundial de la Salud (OMS) y otros especialistas el ruido no modifica el medio ambiente, pero incide en el órgano de percepción fisiológico, el oído; el efecto producido en el órgano de la audición del ser humano por las vibraciones del aire, afecta las actividades del desarrollo social del individuo, como en la comunicación, aprendizaje, concentración, descanso y distorsiona la información.

El término contaminación acústica hace referencia al ruido (entendido como sonido excesivo y molesto), por tanto el ruido es el conjunto de sonidos ambientales nocivos que recibe el oído, por estas características es considerado como un contaminante, es decir, un sonido molesto que puede producir efectos nocivos tanto fisiológicos y psicológicos. Los principales agentes causantes de la contaminación acústica se derivan de la actividad humana como el transporte, la construcción, la industria, los locales públicos, entre otros. Si el ruido excede los límites previstos por organismos especializados, se corre el riesgo de una disminución importante en la capacidad auditiva, así como la posibilidad de trastornos que van desde lo psicológico (paranoia, perversión) hasta lo sexual (impotencia).

\section{REFERENCIAS NORMATIVAS}

Las normas técnicas y legales brindan las pautas para la evaluación del ruido en diversos escenarios como centros laborales y urbanos, facilitan el componente técnico, los procedimientos para disminuir o silenciar. El Comité Técnico de Acústica, de la Organización Internacional de Normalización (ISO, por sus siglas en inglés), ha emitido más de cien normas vinculadas con el ruido; por cierto es una evidencia de la trascendencia de este fenómeno a escala mundial.
La Constitución Política del Perú de 1993 establece en el marco general de la política ambiental (artículo $67^{\circ}$ ) que el Estado Peruano determina la política nacional ambiental y promueve el uso sostenible de sus recursos naturales. Asimismo el Código del Medio Ambiente y de los Recursos Naturales (D. L. $N^{\circ}$ 613), establece en la norma décima del título preliminar que las normas relativas a la protección y conservación del medio ambiente y sus recursos son de orden público. Dicho código incorpora en la legislación peruana el principio de contaminadorpagador, principio de prevención, la obligación de presentar estudios de impacto ambiental y la participación ciudadana.

El Decreto Supremo No 085-2003-PCM, Reglamento de Estándares Nacionales de Calidad Ambiental para Ruido, establece en el Artículo 4.- De los Estándares Primarios de Calidad Ambiental para Ruido, los niveles máximos de ruido en el ambiente no deben excederse para proteger la salud humana. Se consideran como parámetro el nivel de presión sonora continuo equivalente con ponderación $A$ (LAeqT) y toma en cuenta zonas de aplicación y horarios, delimitándolo según el cuadro 1.

Las zonas residencial, comercial e industrial son establecidas como tales por la municipalidad correspondiente, para la zona especial se aplican los límites máximos siguientes 50 dBA para el horario diurno y 40 dBA para el horario nocturno. Asimismo en el Artículo 11 se establece que los estándares nacionales de calidad ambiental para ruido constituyen un objetivo de política ambiental y de referencia obligatoria en el diseño y aplicación de las políticas públicas, asimismo se precisa que en tanto el Ministerio de Salud no emita una Norma Nacional para la medición de ruidos y los equipos a utilizar, éstos serán determinados de acuerdo a lo establecido en las Normas Técnicas ISO 19961:1982: Acústica - Descripción y mediciones de ruido ambiental, Parte I: Magnitudes básicas y procedimientos e ISO 1996- 2:1987: Acústica Descripción y mediciones de ruido ambiental, Parte II: Recolección de datos pertinentes al uso de suelo.

Cuadro 1. Estándares nacionales de calidad ambiental para ruido

\begin{tabular}{|l|c|c|}
\hline \multicolumn{1}{|c|}{$\begin{array}{c}\text { ZONAS DE } \\
\text { APLICACIÓN }\end{array}$} & $\begin{array}{c}\text { HORARIO DIURNO } \\
\text { 07:01 a 22:00 horas) }\end{array}$ & $\begin{array}{c}\text { HORARIO NOCTURNO } \\
\text { (22:01 a 07:00 horas) }\end{array}$ \\
\hline $\begin{array}{l}\text { Zona de protección } \\
\text { especial }\end{array}$ & $50 \mathrm{~dB}$ & $40 \mathrm{Db}$ \\
\hline Zona residencial & $60 \mathrm{~dB}$ & $50 \mathrm{Db}$ \\
\hline Zona comercial & $70 \mathrm{~dB}$ & $60 \mathrm{Db}$ \\
\hline
\end{tabular}

Fuente: D.S. 0085-2003-PCM (Anexo No 1) 
De otro lado la Ordenanza $N^{\circ}$ 127-MSI de la Municipalidad de San Isidro, jurisdicción a la cual pertenece la zona elegida, ha reglamentado acerca del área de influencia del Bosque El Olivar, en forma explícita en el Artículo 23.1 menciona que los límites permisibles se basan en los Estándares Nacionales de Calidad Ambiental para Ruido, previstos en el Decreto Supremo No 085-2003-PCM y como sanción por el incumplimiento está previsto en el artículo 24.2 la revocatoria de la Licencia Municipal de Funcionamiento otorgada. No menciona otro tipo de sanción.

Por cierto la norma peruana es más flexible que la norma de organizaciones internacionales como la Comunidad Europea y los valores recomendados por la OMS son 55 y $45 \mathrm{~dB}$ diurno y nocturno para el ámbito exterior, que es lo mismo la zona residencial en la legislación peruana.

\section{METODOLOGÍADE MEDICIÓN}

Para conocer el impacto que origina el ruido se procedió a encuestar tanto a los transeúntes, conductores y usuarios de vehículos, a lo largo de la avenida Javier Prado comprendida entre las avenidas Paseo de la República y Arenales en el horario 07:00-09:00 y 15:00-19:00 horas, la ficha de la encuesta se muestra en el cuadro 2.

\section{RESULTADOS}

Los resultados de la encuesta se muestran en el cuadro 3, de ello se desprende que la mayor parte de

\section{Cuadro 2. Ficha de encuesta}

Buenos días/tardes: Hemos hecho selección al azar y usted ha sido elegido para una encuesta en esta avenida. ¿Le importa contestar unas breves preguntas?

CONTESTA...... NO QUIERE CONTESTAR....... FECHA :.......... DIA:.......... HORA:.............

1. Datos generales: EDAD:........... SEXO:.......

2. ¿Qué grado de molestia le causa el ruido?:

MUCHO..... REGULAR. NADA ...........

3. ¿En cuanto a su salud, el ruido afecta?:

NO......... MAS O MENOS

SI...........

4. ¿Qué ruido le molesta más?

VEHICULOS......... LOCALES PÚBLICOS........

VECINOS ............. OTROS. las personas entrevistadas corresponden al grupo con edades mayores de 51 años $(38.46 \%)$, seguido por el grupo con edades comprendidas entre 36 y 51 años $(28.46 \%)$ y el grupo con edades comprendidas entre 26 y 35 años (20.77\%).

Un $57.69 \%$ de las personas encuestadas han resultado ser hombres y $42.31 \%$ mujeres. Un $12.31 \%$ de las personas entrevistadas ha manifestado que el ruido ambiental que percibían no le molesta "nada". Un $26.15 \%$ de la muestra ha declarado estar "algo" molesto por el ruido. Finalmente, un $61.54 \%$ de las personas encuestadas ha expresado que estaba "muy" molesto a causa del ruido.

De acuerdo con las respuestas, el efecto del ruido en un $21.15 \%$ de los encuestados no afectaba, en el $32.69 \%$ más o menos y si afectaba al $46.15 \%$ restante.

Las fuentes de ruido que resultan más molestias para las personas entrevistadas son, por orden decreciente, los vehículos $(62.69 \%)$, lugares públicos $(23.46 \%)$ y los vecinos $(3.85 \%)$, en cuanto a otros no respondieron.

En las figuras 1, 2 y 3 se muestran las manifestaciones de los encuestados y su reacción al problema.

\section{PROPUESTAS PARA AMENGUAR LA CONTAMINACIÓN}

Los resultados evidencian que en las horas pico la presión del ruido influye en la reacción de las

\begin{tabular}{|c|c|c|c|c|c|}
\hline & Cuadro & 3. Resultad & de la & yes & \\
\hline Pregunta & & & Datos & & \\
\hline 1 & Edades & Masculino & Femenino & & Total \\
\hline & $15-25$ & 20 & 12 & & 32 \\
\hline & $26-35$ & 32 & 22 & & 54 \\
\hline & $36-50$ & 45 & 29 & & 74 \\
\hline & $\begin{array}{c}\text { más de } \\
51\end{array}$ & 53 & 47 & & 100 \\
\hline & Total & 150 & 110 & & 260 \\
\hline 2 & & Grado de molestia & & & \\
\hline & Mucho & Regular & Nada & & Total \\
\hline & 120 & 85 & 55 & & 260 \\
\hline 3 & & $\begin{array}{c}\text { Afecta el ruido en } \\
\text { la salud }\end{array}$ & & & \\
\hline & No & Más o menos & $\mathrm{Si}$ & & Total \\
\hline & 32 & 68 & 160 & & 260 \\
\hline 4 & & Ruido que molesta & & & \\
\hline & Vehículos & Lugares públicos & Vecinos & Otros & Total \\
\hline & 163 & 61 & 36 & 0 & 260 \\
\hline
\end{tabular}

Fuente: Elaboración propia 
Diagrama 1. Grado de molestia del ruido

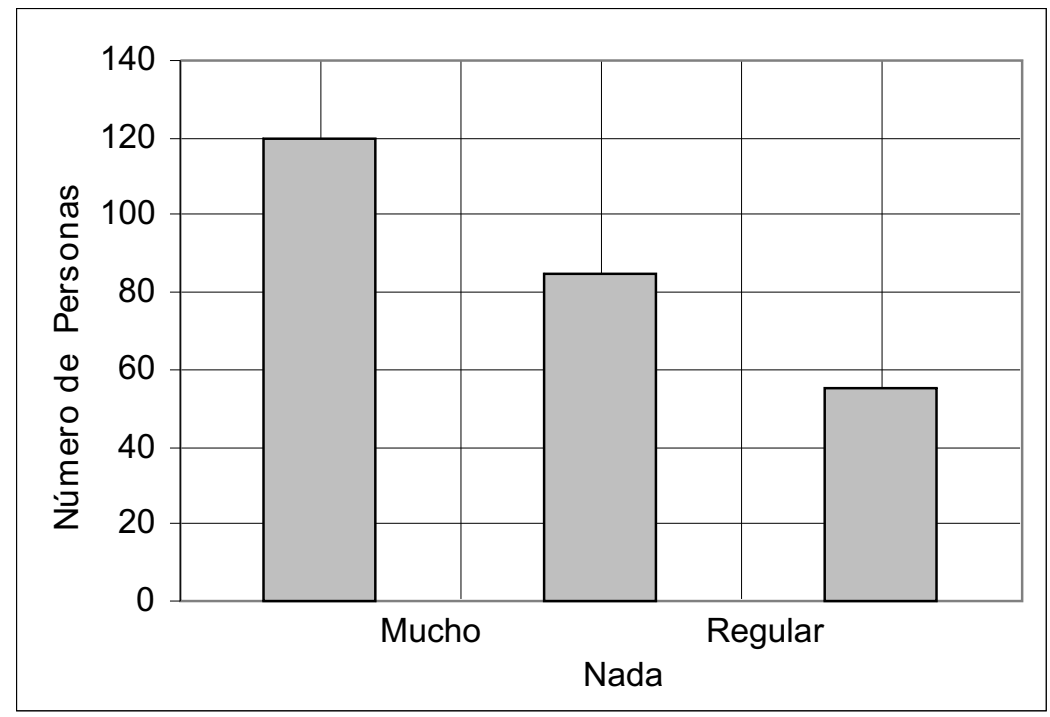

Fuente: Elaboración propia

Diagrama 2. Efecto del ruido en la salud

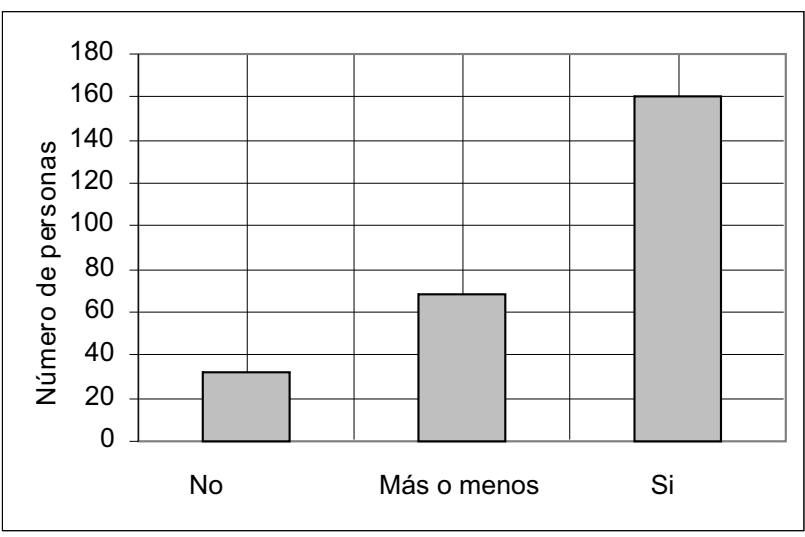

Fuente: Elaboración propia

personas, situación que compete a las autoridades adoptar medidas correctivas.

En este sentido, se proponen las siguientes acciones:

- Realizar el diseño medioambiental óptimo de las vías de comunicación, complementado con una planificación compatible del uso del suelo alrededor de las vías.

- Si bien el nivel sonoro continuo aumenta con la velocidad del tráfico y con la intensidad del mismo, se debe considerar también la presencia de obstáculos en la trayectoria de propagación, por lo tanto se deben mejorar o implementar medidas como limitar y hacer cumplir la velocidad media del tráfico vehicular y distribuirlo por otras vías ofreciendo itinerarios de menor costo, de ese modo
Diagrama 3. Agentes sonoras que mas molestan

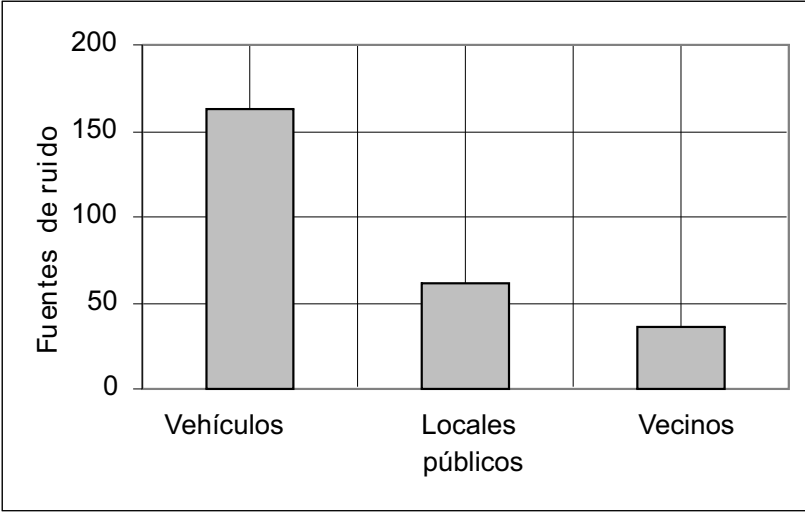

Fuente: Elaboración propia

reducir la intensidad de tráfico en determinados tramos.

- Mejorar la fluidez del tráfico, estableciendo paraderos para vehículos de servicio público en forma alternada y cada cierta distancia para evitar la congestión, así como controlar y limitar los gritos desesperados de los cobradores por conseguir pasajeros.

- Implementar con la cobertura vegetal la berma central de la avenida Javier Prado con plantas de rápido crecimiento y abundante follaje como ficus, sauces, álamos y otros; a modo de ejemplo las palmeras plantadas en las cuadras 8 al 15 de la misma avenida están demorando en alcanzar su desarrollo y, a la vez se desprenden rápidamente 
de sus hojas, por tanto poca aptitud para amortiguar el ruido.

- Controlar a los conductores de los vehículos que utilizan sus radios a volumen alto y los claxon en forma innecesaria, en algunos vehículos utilizan sirena como claxon que aumenta el nivel de ruido y causa molestia. Así como el mantenimiento oportuno y adecuado de los vehículos, siendo conveniente emplear silenciadores apropiados.

- Construir puentes peatonales en las intersecciones con Las Palmeras. Las Flores con Eucaliptos, Los Naranjos, avenidas Iquitos, Rivera NavarreteMagnolias, Petit Thouars, Arenales, de ese modo habría mayor fluidez en la circulación vehicular, con ello bajar el nivel de uso de medios como claxon al desesperarse el conductor ante tantas paradas como intersecciones haya y por el cruce temerario de los transeúntes.

\section{CONCLUSIONES}

Las personas encuestadas han respondido que los vehículos son los que causan más molestia con el ruido, seguido de los locales públicos y en menor proporción los vecinos, no existen paraderos para vehículos de servicio público, esto permite que se genere caos en el tránsito con ello se incrementa el nivel de ruido.

\section{REFERENCIAS BIBLIOGRÁFICAS}

1. Marzinzik, M. y Kollmeier, B. (2003) Predicting the Subjective Quality of Noise Reduction Algorithms for Hearing Aids, Acta Acustica, vol. 89, pp. 521529, Alemania.

2. Torres, P. (17/07/2006) No una sino varias Javier Prado, Diario El Comercio, pág. 27, Lima, Perú.

3. Varga A. y H. Steeneken, H. (1993) Assessment for Automatic Speech Recognition: II. NOISEX-92: A Database and an Experiment to Study the Effect of Additive Noise on Speech Recognition Systems, Speech Communication, vol. 12, No 3, pp. 247251, USA.

4. Vaseghi, S. V.(2000)Advanced Digital Signal Processing and Noise Reduction, 2nd Edition, John Wiley \& Sons, USA. 